Check for updates

Cite this: Chem. Commun., 2018, 54,2755

Received 23rd November 2017, Accepted 16th February 2018

DOI: $10.1039 / c 7 c c 09017 j$

rsc.li/chemcomm

\section{One-step synthesis of 2D-layered carbon wrapped transition metal nitrides from transition metal carbides (MXenes) for supercapacitors with ultrahigh cycling stability $\dagger$}

\author{
Wenyu Yuan, (D ${ }^{a}$ Laifei Cheng, ${ }^{\star a}$ Heng Wu, ${ }^{a}$ Yani Zhang, ${ }^{a}$ Shilin Lv ${ }^{a}$ and \\ Xiaohui Guo*b
}

A novel one-step method to synthesize 2D carbon wrapped TiN (CATiN) was proposed via using 2D metal carbides (MXenes) as precursors. This study provides a novel approach to synthesize carbon wrapped metal nitrides.

Supercapacitors (SCs) are considered to be one of the candidates for future energy storage devices owing to their high power density and long cycling life. ${ }^{1,2}$ Recently, great efforts have been focused on high-rate performance supercapacitors, which can work under high current densities. Transition metal nitrides (TMNs), including TiN, VN, MoN, $\mathrm{Mo}_{2} \mathrm{~N}, \mathrm{Ni}_{3} \mathrm{~N}$, etc., have been considered as promising candidates for high-rate SCs due to their high electrical conductivities $\left(\sim 10^{4} \mathrm{~S} \mathrm{~cm}^{-1}\right)$ and high thermal stability, and have been used widely as electrodes in SCs. ${ }^{3-7}$ TiN, with a high electrical conductivity of $5.5 \times 10^{4} \mathrm{~S} \mathrm{~cm}^{-1}$, fits well with the requirements for high-rate performance supercapacitors. $^{8,9}$ However, TiN suffers from poor electrochemical stability because of the generation of transition metal oxide layers in electrolytes, deteriorating the activity and resulting in poor stability and low specific capacitance for SCs. ${ }^{10-12}$ To improve the cyclic stability and activity, the use of a carbon wrapped structure, which can effectively prevent its electrochemical oxidation, was proved to be an efficient method and has received growing attention. ${ }^{13}$ Lei et al. designed a selfsupported carbon coated TiN nanotube array to improve the cycling stability. ${ }^{14}$ Mai et al. recently reported TiN@C nanotubebased fiber electrodes through nitridation and a carbon coating process. ${ }^{15}$ A relatively high cyclic stability of $\sim 80 \%$ capacitance retention after 10000 cycles was achieved. However, the to-datereported method for the synthesis of C@TMN structures mainly involves two steps: (1) the synthesis of metal nitrides and (2) the deposition of carbon layers. It is of critical significance to develop

\footnotetext{
${ }^{a}$ Science and Technology on Thermostructural Composite Materials Laboratory, Northwestern Polytechnical University, 710072, Xi'an, P. R. China.

E-mail: chenglf@nwpu.edu.cn

${ }^{b}$ College of Chemistry and Materials Science, Northwest University, Xi'an 710069,

P. R. China.E-mail: guoxh2009@nwu.edu.cn

$\dagger$ Electronic supplementary information (ESI) available. See DOI: 10.1039/c7cc09017j
}

an efficient, simple process for carbon wrapped metal nitrides to replace the present complex method.

Furthermore, the architecture usually affects the resulting performance. To date, 1D C@TMN architectures, including nanotubes, ${ }^{14}$ nanoarrays, ${ }^{5}$ nanowires, ${ }^{16}$ etc., have been mostly discussed. However, to the best of our knowledge, no studies on 2D carbon wrapped TMN structures have been reported because it is very hard for TMNs to form 2D layered structures via the traditional exfoliation strategy. ${ }^{17} \mathrm{~A} 2 \mathrm{D}$ layered structure is always regarded to have a fast ion transport rate and large SSA, beneficial to the surface chemical reactions and the absorption-desorption process. ${ }^{18,19}$ Previous works demonstrated that $2 \mathrm{D}$ nanostructures can be successfully synthesized via the chemical conversion of $2 \mathrm{D}$ precursors. ${ }^{20,21}$ To synthesize a $2 \mathrm{D}$ layered C@TMN structure, 2D transition metal carbides (MXenes) with their excellent properties in energy storage and conversion fields, which recently received much attention, ${ }^{22-24}$ were chosen as precursors. Thus, we developed a novel synthesis method to drive metal carbides into a C@TiN structure via one-step nitridation of 2D carbides. To the best of our knowledge, no studies have been reported on MXene-derived TMNs to date. The in situ generated carbon layers not only enhance the charge transfer efficiency, but also can effectively prevent the oxidation of metal nitrides in electrolytes, resulting in improved specific capacitance, rate capacitance and cycling stability. This universal approach is promising to be applied to synthesize more carbon wrapped TMNs for energy storage and conversion fields.

The one-step synthetic procedure for C@TiN is illustrated in Fig. 1a. After one-step nitridation, the TiN nanosheets wrapped by the carbon layers were produced, meanwhile the $2 \mathrm{D}$-layered structure could be well preserved. The layered structure has been well preserved during the nitridation process (Fig. 1b and c). The thickness of the C@TiN layer is $\sim 20 \mathrm{~nm}$. The total nitridation mechanism is illustrated in Fig. 1d. $\mathrm{NH}_{3}$ molecules can destroy the $\mathrm{Ti}-\mathrm{C}$ bonds, followed by the formation of $\mathrm{Ti}-\mathrm{N}$ bonds. The carbon atoms can react with hydrogen atoms in $\mathrm{NH}_{3}$ molecules, and generate $\mathrm{CH}_{4}$ molecules. Then $\mathrm{CH}_{4}$ molecules are decomposed into carbon atoms and hydrogen atoms 
(a)
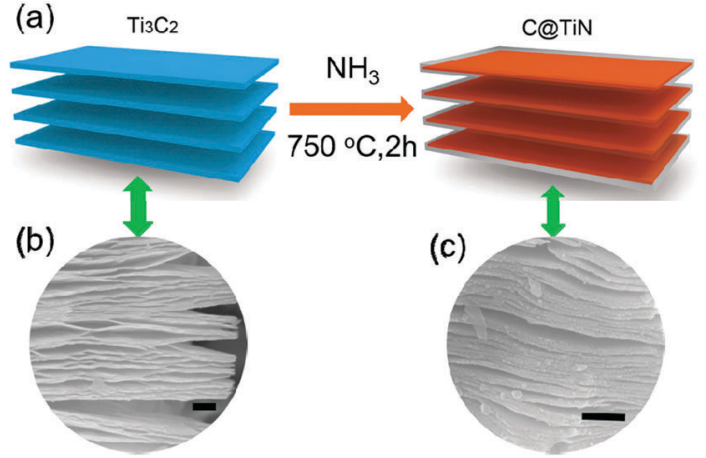

(d)
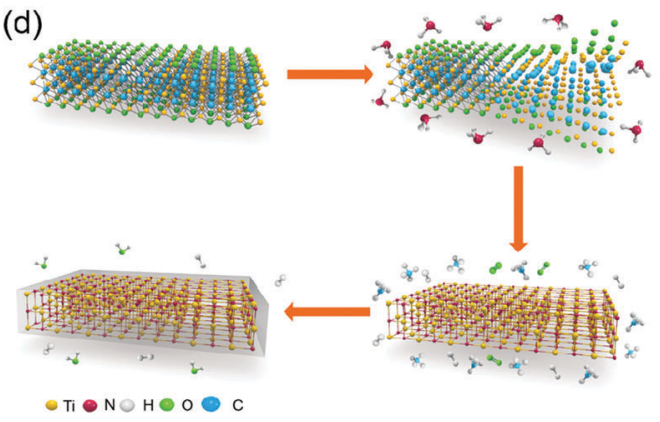

Fig. 1 (a) Schematic diagram of the synthesis process for CaTiN. (b) The morphology of $\mathrm{Ti}_{3} \mathrm{C}_{2}$. (c) The morphology of the obtained C@TiN. The bar in (b) and (c) is $1 \mu \mathrm{m}$. (d) Schematic of the structural transformation from $\mathrm{Ti}_{3} \mathrm{C}_{2}$ to $\mathrm{C} @ \mathrm{aTiN}$ during the calcination at $750{ }^{\circ} \mathrm{C}$ in an $\mathrm{NH}_{3}$ atmosphere.

at high temperature. The generated carbon atoms are deposited on the surface of TiN nanosheets, resulting in a 2D-layered C@TiN structure, meanwhile some of the hydrogen was oxidized to produce $\mathrm{H}_{2} \mathrm{O}$ molecules. These reaction processes can happen simultaneously during the nitridation process, thus realizing the one-step synthesis of 2D-layered C@TiN hybrids from 2D metal carbides (MXenes). The total reaction equation can be summarized as the following:

$$
\mathrm{Ti}_{3} \mathrm{C}_{2} \mathrm{O}_{2}+3 \mathrm{NH}_{3} \rightarrow 3 \mathrm{TiN}+2 \mathrm{C}+2 \mathrm{H}_{2} \mathrm{O} \uparrow+5 / 2 \mathrm{H}_{2} \uparrow
$$

The 2D layered structure was further identified using TEM, as shown in Fig. 2a. A 2D C@TiN nanosheet can be clearly observed. The HRTEM image further clearly shows that the well-crystallized TiN (inset in Fig. 2b) is wrapped by a layer of amorphous carbon with a thickness of $\sim 2-3 \mathrm{~nm}$. The HAADFSTEM image (Fig. S1, ESI $\dagger$ ) clearly shows a carbon shell at the surface of the TiN. The EDX mapping further suggests a carbon wrapped TiN structure. XRD was further carried out to analyze the phase transformation during the nitridation process, as shown in Fig. 2c. The (002) characteristic peak of $\mathrm{Ti}_{3} \mathrm{C}_{2}$, located at $9.01^{\circ}$, indicates the successful synthesis of $\mathrm{Ti}_{3} \mathrm{C}_{2}$ MXenes. ${ }^{24}$ After calcination under an $\mathrm{NH}_{3}$ atmosphere, the $\mathrm{Ti}_{3} \mathrm{C}_{2}$ MXenes were totally transformed into TiN. The main crystalline phase of C@TiN can be assigned to the TiN phase (JCPDS: 38-1420), in which the main diffraction peaks are at $36.8^{\circ}$ (111), $42.7^{\circ}$ (200), and $62.0^{\circ}(220)$, which are in line with the pure TiN. ${ }^{25}$ Considering that the amorphous carbon cannot be identified
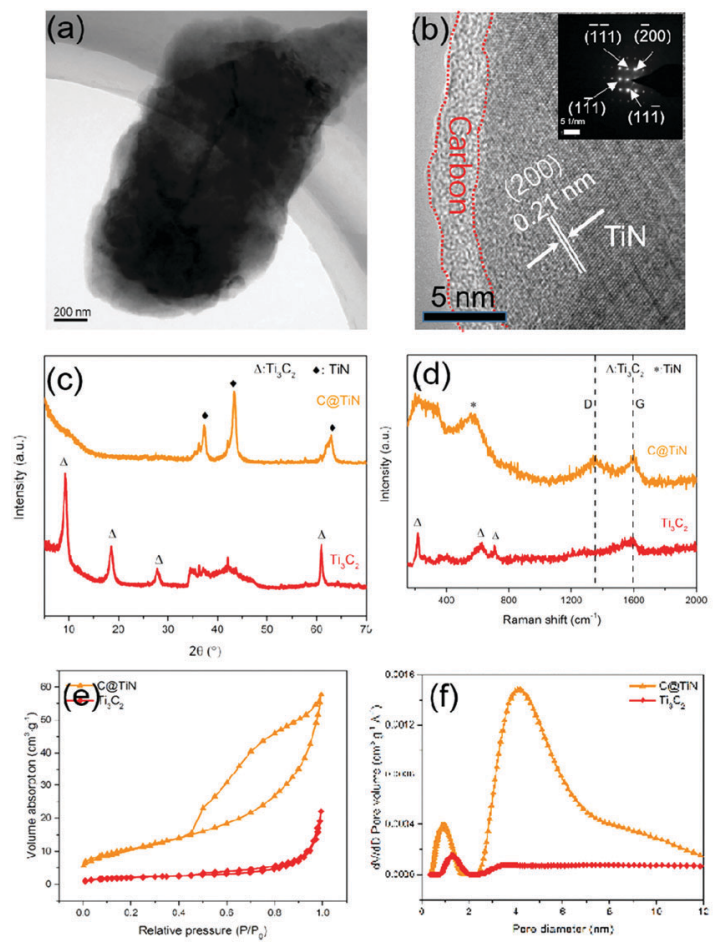

Fig. 2 (a) The TEM of CATiN. (b) HRTEM of CaTiN. The inset is the SAED of TiN. (c-f) The XRD, Raman spectra, $\mathrm{N}_{2}$ absorption/desorption isotherm and pore size distribution of $\mathrm{Ti}_{3} \mathrm{C}_{2}$ and CATiN.

using XRD, the Raman spectra were further recorded to detect the amorphous carbon. The two peaks at $1352 \mathrm{~cm}^{-1}$ (D band) and $1583 \mathrm{~cm}^{-1}$ (G band) are assigned to the defects and the in-plane vibration of $\mathrm{sp}^{2}$ carbon atoms, indicating the existence of carbon. ${ }^{26,27}$ The $I_{\mathrm{D}} / I_{\mathrm{G}}$ value reached 0.79 , suggesting that the carbon was amorphous. ${ }^{28}$ The band at $557 \mathrm{~cm}^{-1}$ and the broadened band at $200-300 \mathrm{~cm}^{-1}$ are caused by TiN. ${ }^{29,30}$ Thus, we could confirm that $\mathrm{Ti}_{3} \mathrm{C}_{2}$ MXenes have been totally transformed into TiN and carbon after the $\mathrm{NH}_{3}$ thermal treatment at high temperature. Besides $\mathrm{Ti}_{3} \mathrm{C}_{2}$ MXenes, more $2 \mathrm{D}$ metal carbide MXenes can be used to synthesize carbon wrapped metal nitrides. The total reaction equation can be concluded as the following:

$$
\mathrm{M}_{x} \mathrm{C}_{y} \mathrm{O}_{2}+x \mathrm{NH}_{3} \rightarrow x \mathrm{MN}+y \mathrm{C}+2 \mathrm{H}_{2} \mathrm{O} \uparrow+\frac{3 x-4}{2} \mathrm{H}_{2} \uparrow
$$

The $\mathrm{N}_{2}$ adsorption-desorption isotherms of C@TiN are shown in Fig. 2e. ${ }^{31}$ Compared with $\mathrm{Ti}_{3} \mathrm{C}_{2}\left(7.96 \mathrm{~m}^{2} \mathrm{~g}^{-1}\right)$, the SSA of 2D-layered C@TiN was significantly improved, it was up to $63.00 \mathrm{~m}^{2} \mathrm{~g}^{-1}$. Moreover, the C@TiN shows a unique hierarchically porous structure (Fig. 2f). There are numerous micropores $(1.1 \%)$, mesopores $(73.3 \%)$, and macropores $(25.6 \%)$ within the C@TiN hybrids. The high percentage of mesopores can efficiently promote the transport of ions and electrons. ${ }^{32,33}$ The pore size distribution of the 2D-layered C@TiN hybrids was mainly focused around 1.3 and $4.1 \mathrm{~nm}$, as shown in Fig. 2f.

The chemical components and elemental chemical state were further characterized using XPS and are displayed in Fig. S2 (ESI $\dagger$ ). The elements $\mathrm{O}, \mathrm{Ti}, \mathrm{N}$, and $\mathrm{C}$ can be clearly identified in the survey XPS spectra, as shown in Fig. S2a (ESI†). The oxygen in 
C@TiN is caused by the functional groups on the surface of MXene precursors. The high-resolution C1s electron XPS spectra are displayed in Fig. $\mathrm{S} 2 \mathrm{~b}$ (ESI $\dagger$ ). The peak at $281.9 \mathrm{eV}$ in the $\mathrm{Ti}_{3} \mathrm{C}_{2}$ precursors (Fig. S3, ESI $\dagger$ ), which is assigned to the $\mathrm{C}-\mathrm{Ti}$ bond, disappeared after nitridation, indicating that the $\mathrm{C}-\mathrm{Ti}$ bond has been totally destroyed. ${ }^{34}$ The peak at $284.5 \mathrm{eV}$ is corresponding to the $\mathrm{C}-\mathrm{C}$ bond. Some functional groups exist in the carbon layers owing to the peak at $286.2 \mathrm{eV}(\mathrm{C}-\mathrm{O}$ bond). The peaks at $285.7 \mathrm{eV}$ and $288.2 \mathrm{eV}$ are assigned to the $\mathrm{C}-\mathrm{N}$ and $\mathrm{C}-\mathrm{N}-\mathrm{Ti}$ bonds, respectively. ${ }^{35}$ The three main peaks at 396.3, 398.4, and $401.0 \mathrm{eV}$ are assigned to the $\mathrm{N}-\mathrm{Ti}, \mathrm{N}-\mathrm{C}$, and $\mathrm{C}-\mathrm{N}-\mathrm{Ti}$ bonds, respectively. ${ }^{17}$ The chemical bonds between carbon and TiN can lead to strong integration, which is helpful for the stability. The high-resolution Ti2p electron XPS spectra further suggest the successful synthesis of TiN. ${ }^{6,36}$

The electrochemical performances of C@TiN, $\mathrm{Ti}_{3} \mathrm{C}_{2}$ and $\mathrm{TiN}$ were evaluated using $C V$, galvanostatic charge-discharge (GCD), and EIS techniques in a three-electrode system using $2 \mathrm{M} \mathrm{KOH}$ as the electrolyte. The effects of calcination conditions on the electrochemical performance are discussed here. Fig. 3a shows the $C V$ curves of these samples at a scan rate of $10 \mathrm{mV} \mathrm{s}^{-1}$. All samples delivered rectangular shaped curves for typical electrochemical double layer capacitance (EDLC) performance. Among the four C@TiN samples, C@TiN-750-2 delivered the highest electrochemical performance owing to the high SSA, high pore volume and high ratio of mesopores (Table S1, ESI $\dagger$ ). C@TiN calcinated at $850{ }^{\circ} \mathrm{C}$ suffers from heavy aggregation (Fig. S4, ESI $\dagger$ ), which leads to the low SSA and pore volume. Furthermore, the $C V$ curves of C@TiN-750-2 under various scan rates have been studied and are shown in Fig. S5a (ESI $\dagger$ ). We found that even under a high scan rate of $500 \mathrm{mV} \mathrm{s}^{-1}$, the $C V$ curve did not obviously change, indicating a high rate capacitance. Fig. $3 \mathrm{~b}$ shows the GCD profiles of C@TiN, TiN and $\mathrm{Ti}_{3} \mathrm{C}_{2}$, at a current density of $1 \mathrm{~A} \mathrm{~g}^{-1}$. Similar to the results in Fig. 3a, C@TiN-750-2 shows the longest discharge time with a low IR drop of $0.0053 \mathrm{~V}$ among all samples. The GCD profiles of C@TiN-750-2 measured
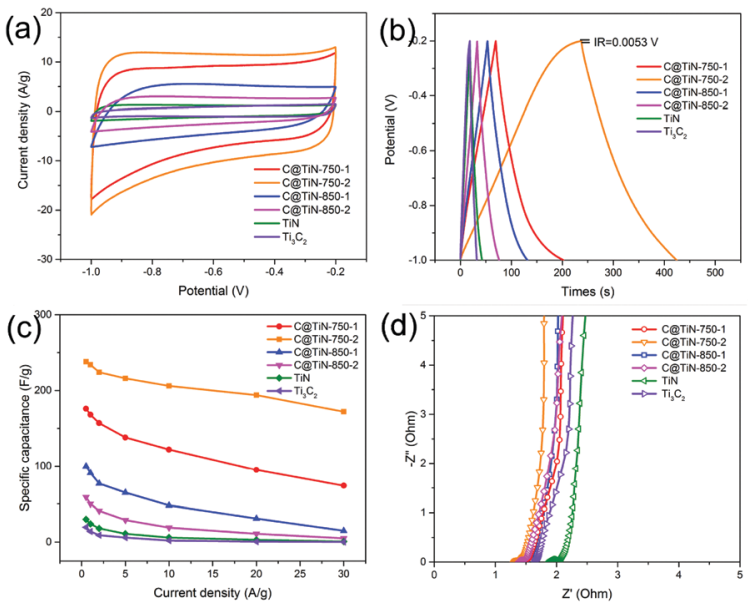

Fig. 3 The CV curves at a scan rate of $100 \mathrm{mV} \mathrm{s}^{-1}$ (a), GCD curves under a current density of $1 \mathrm{~A} \mathrm{~g}^{-1}$ (b), the specific capacitance vs. current density (c), and Nyquist plots of CaTiN-750-1, CaTiN-750-2, CaTiN-850-1, CaTiN-850-2, $\mathrm{TiN}$ and $\mathrm{Ti}_{3} \mathrm{C}_{2}$, respectively. at the current density ranging from 0.5 to $30 \mathrm{~A} \mathrm{~g}^{-1}$ are shown in Fig. S5b (ESI $\dagger$ ). Interestingly, the IR drop did not obviously increase even under a high current density of $20 \mathrm{~A} \mathrm{~g}^{-1}$, suggesting a high rate performance. The gravimetric specific capacitances of 6 samples at different current densities were calculated from the corresponding discharge curves, and the results are shown in Fig. 3c. The specific capacitances of C@TiN-750-1, C@TiN-750-2, C@TiN-850-1, C@TiN-850-2, TiN, and $\mathrm{Ti}_{3} \mathrm{C}_{2}$ under a current density of $0.5 \mathrm{~A} \mathrm{~g}^{-1}$ are 176, 238, 100, 59, 30, and $20 \mathrm{~F} \mathrm{~g}^{-1}$, respectively. The specific capacitance retention of C@TiN-750-2 under a high current density of $30 \mathrm{~A} \mathrm{~g}^{-1}$ reached $72.3 \%$, which is much higher than those of C@TiN-750-1 (41.9\%), C@TiN-850-1 (15.0\%), C@TiN-850-2 (9.0\%), TiN (2.5\%) and $\mathrm{Ti}_{3} \mathrm{C}_{2}$ (1.2\%). Fig. 3d shows the Nyquist plots of the 6 samples. C@TiN-750-2 possessed the largest slope among all samples due to the high ratio of mesopores. C@TiN-750-2 shows the lowest equivalent series resistance (ESR) of $1.28 \Omega$ and a low charge transfer resistance of 0.18 $\Omega$, suggesting a high electrical conductivity of C@TiN-750-2.

The cycling performance of the C@TiN samples was also studied via cyclic GCD tests under a current density of $2 \mathrm{~A} \mathrm{~g}^{-1}$, as shown in Fig. 4a. The specific capacitance of bare TiN sharply decreased during the beginning 2000 cycles owing to the oxidation of TiN via the following equation: ${ }^{13}$

$$
\mathrm{TiN}+2 \mathrm{H}_{2} \mathrm{O} \rightarrow \mathrm{TiO}_{2}+1 / 2 \mathrm{~N}_{2}+4 \mathrm{H}^{+}+4 \mathrm{e}^{-} .
$$

The capacitance retention after 10000 cycles is only $29.2 \%$. The C@TiN samples showed much better stability in electrolytes. C@TiN-750-2 delivered the highest cyclic stability with an ultrahigh capacitance retention of $93.1 \%$. Its specific capacitance and cycling stability are superior to most reported TiN electrodes. Furthermore, we investigated the $C V$ curves, the Nyquist plots and the XPS Ti2p spectra after 10000 cycles, as shown in Fig. 4b-d. The rectangular shaped $C V$ curve after 10000 cycles suggested a typical EDLC performance, indicating that the structure has not changed obviously. The ESR and the charge transfer resistance
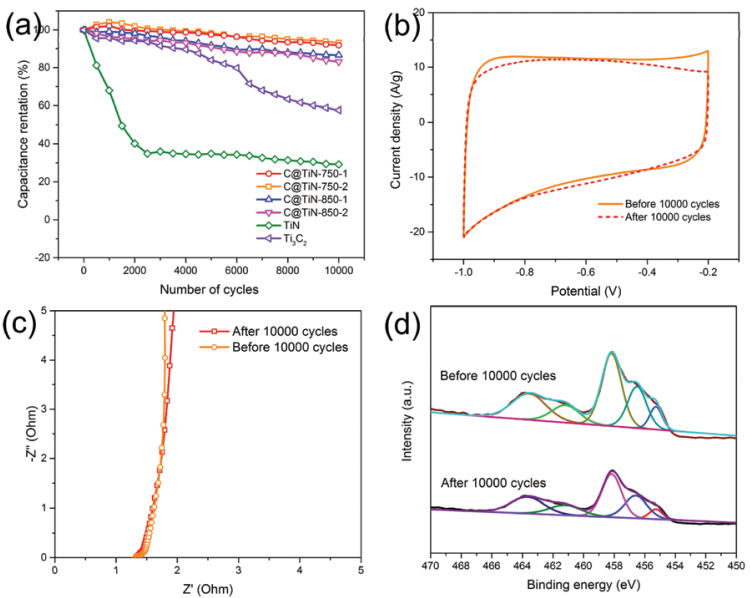

Fig. 4 (a) Plots of capacitance retention vs. number of cycles under a current density of $2 \mathrm{~A} \mathrm{~g}^{-1}$. (b) The CV curves of C (aTiN-750-2 at a scan rate of $100 \mathrm{mV} \mathrm{s}^{-1}$ before and after 10000 cycles. (c) The Nyquist plots of CaTiN-750-2 before and after 10000 cycles. (d) The XPS Ti2p spectra of CaTiN-750-2 before and after 10000 cycles. 
almost remained the same, meanwhile the slope at the lowfrequency region slightly decreased. The XPS Ti2p spectra suggest that no obvious intensity enhancement of the Ti-O bond was observed after cyclic tests and the main bonds have been well preserved. No obvious oxidation occurs during the electrochemical process. These results suggested that the carbon could significantly improve the cyclic stability of TiN in electrolytes. Two reasons are responsible for the high cyclic stability of C@TiN: (1) the carbon layers can effectively prevent the electrochemical oxidation reaction of TiN and (2) the carbon shell acts as a cage for TiN and prevents the structural damage and mass loss during cycling.

To evaluate the energy and power density, a solid-state supercapacitor was prepared using two parallel C@TiN samples as electrodes and $\mathrm{KOH} / \mathrm{PVA}$ gel as the electrolyte. Fig. S6a (ESI $\dagger$ ) shows that the shape of $C V$ curves does not change under different potential windows, indicating that the supercapacitor can be operated under wide operating potential windows. The rectangular shaped $C V$ curve under an ultrahigh scan rate of $2000 \mathrm{mV} \mathrm{s}^{-1}$ suggests a high-rate performance. The GCD curves are shown in Fig. S6c (ESI $\dagger$ ) and the calculated specific capacitances of the SCs are 61.2, 56.9, 53.0, 52.1, and $51.3 \mathrm{~F} \mathrm{~g}^{-1}$, respectively. The Ragone plots of the C@TiN supercapacitors derived from the GCD curves are displayed in Fig. S6d (ESI $\dagger$ ). The energy density is $5.46 \mathrm{~W} \mathrm{~h} \mathrm{~kg}^{-1}$ at a power density of $111 \mathrm{~W} \mathrm{~kg}$ based on the total mass of two electrodes. The energy density still remained at $4.56 \mathrm{~W} \mathrm{~h} \mathrm{~kg}^{-1}$ at a high power density of $2161 \mathrm{~W} \mathrm{~kg}^{-1}$. The power density increased $\sim 2000 \%$, and the energy density only decreased $\sim 16 \%$, suggesting high-rate performance and high stability under high current densities. Table S2 (ESI $\dagger$ ) compares the electrochemical performances of the synthesized C@TiN and reported TiN-based electrodes, and the C@TiN in this work is superior to the reported TiN-based SC materials.

In summary, we delivered a novel one-step approach to synthesize 2D-layered carbon wrapped TMNs for supercapacitors. The optimal specific capacitance reached $238 \mathrm{~F} \mathrm{~g}^{-1}$ under the current density of $0.5 \mathrm{~A} \mathrm{~g}^{-1}$. Even under a current density of $30 \mathrm{~A} \mathrm{~g}^{-1}$, the capacitance retention was still as high as $72.3 \%$, indicating a high rate capacitance. Furthermore, owing to the existence of carbon layers, the C@TiN delivered an ultrahigh cyclic stability (high capacitance retention of $93.1 \%$ after 10000 cycles under the current density of $2 \mathrm{~A} \mathrm{~g}^{-1}$ ). This novel one-step method can be further applied to synthesize other carbon wrapped TMNs and the 2D layered carbon wrapped TMNs are potential candidates for other energy storage and conversion fields.

The authors would like to acknowledge the financial support provided by the National Key R\&D Program of China (No. 2017YFB1103500), the National Natural Science Foundation of China (No. 51302220, 51672218 and 51632007), Northwestern Polytechnical University (NPU) Foundation for Fundamental Research (No. 3102015ZY086), and the Fund of the State Key Laboratory of Solidification Processing in NPU (No. SKLSP201613).

\section{Conflicts of interest}

There are no conflicts to declare.

\section{Notes and references}

1 G. Wang, L. Zhang and J. Zhang, Chem. Soc. Rev., 2012, 41, 797-828.

2 M. Salanne, B. Rotenberg, K. Naoi, K. Kaneko, P.-L. Taberna, C. P. Grey, B. Dunn and P. Simon, Nat. Energy, 2016, 1, 16070.

3 M.-S. Balogun, W. Qiu, W. Wang, P. Fang, X. Lu and Y. Tong, J. Mater. Chem. A, 2015, 3, 1364-1387.

4 D. R. Deng, F. Xue, Y. J. Jia, J. C. Ye, C. D. Bai, M. S. Zheng and Q. F. Dong, ACS Nano, 2017, 11, 6031-6039.

5 P. Yang, D. Chao, C. Zhu, X. Xia, Y. Zhang, X. Wang, P. Sun, B. K. Tay, Z. X. Shen, W. Mai and H. J. Fan, Adv. Sci., 2016, 3, 1500299.

6 X. Lu, G. Wang, T. Zhai, M. Yu, S. Xie, Y. Ling, C. Liang, Y. Tong and Y. Li, Nano Lett., 2012, 12, 5376-5381.

7 S. Yang, J. Kim, Y. J. Tak, A. Soon and H. Lee, Angew. Chem., Int. Ed., 2016, 55, 2058-2062.

8 Z. Cui, C. Zu, W. Zhou, A. Manthiram and J. B. Goodenough, Adv. Mater., 2016, 28, 6926-6931.

9 Z. Li, J. Zhang, B. Y. Guan and X. W. Lou, Angew. Chem., Int. Ed., 2017, 56, 16003-16007.

10 B. Avasarala and P. Haldar, Electrochim. Acta, 2010, 55, 9024-9034.

11 B. M. Gray, A. L. Hector, M. Jura, J. R. Owen and J. Whittam, J. Mater. Chem. A, 2017, 5, 4550-4559.

12 B. Avasarala and P. Haldar, Int. J. Hydrogen Energy, 2011, 36, 3965-3974.

13 X. Lu, T. Liu, T. Zhai, G. Wang, M. Yu, S. Xie, Y. Ling, C. Liang, Y. Tong and Y. Li, Adv. Energy Mater., 2014, 4, 1300994.

14 F. Grote, H. Zhao and Y. Lei, J. Mater. Chem. A, 2015, 3, 3465-3470.

15 P. Sun, R. Lin, Z. Wang, M. Qiu, Z. Chai, B. Zhang, H. Meng, S. Tan, C. Zhao and W. Mai, Nano Energy, 2017, 31, 432-440.

16 Y. Han, X. Yue, Y. Jin, X. Huang and P. K. Shen, J. Mater. Chem. A, 2016, 4, 3673-3677.

17 X. Xiao, H. Yu, H. Jin, M. Wu, Y. Fang, J. Sun, Z. Hu, T. Li, J. Wu, L. Huang, Y. Gogotsi and J. Zhou, ACS Nano, 2017, 11, 2180-2186.

18 E. Pomerantseva and Y. Gogotsi, Nat. Energy, 2017, 2, 17089.

19 R. Mas-Balleste, C. Gomez-Navarro, J. Gomez-Herrero and F. Zamora, Nanoscale, 2011, 3, 20-30.

20 P. Huang, C. Lethien, S. Pinaud, K. Brousse, R. Laloo, V. Turq, M. Respaud, A. Demortière, B. Daffos, P. L. Taberna, B. Chaudret, Y. Gogotsi and P. Simon, Science, 2016, 351, 691-695.

21 C. Liu, S. Zhao, Y. Lu, Y. Chang, D. Xu, Q. Wang, Z. Dai, J. Bao and M. Han, Small, 2017, 13, 1603494.

22 B. Anasori, M. R. Lukatskaya and Y. Gogotsi, Nat. Rev. Mater., 2017, 2, 16098.

23 M. R. Lukatskaya, O. Mashtalir, C. E. Ren, Y. Dall'Agnese, P. Rozier, P. L. Taberna, M. Naguib, P. Simon, M. W. Barsoum and Y. Gogotsi, Science, 2013, 341, 1502-1505.

24 M. Naguib, V. N. Mochalin, M. W. Barsoum and Y. Gogotsi, Adv. Mater., 2014, 26, 992-1005.

25 A. Achour, M. Chaker, H. Achour, A. Arman, M. Islam, M. Mardani, M. Boujtita, L. Le Brizoual, M. Djouadi and T. Brousse, J. Power Sources, 2017, 359, 349-354.

26 A. C. Ferrari, J. Meyer, V. Scardaci, C. Casiraghi, M. Lazzeri, F. Mauri, S. Piscanec, D. Jiang, K. Novoselov and S. Roth, Phys. Rev. Lett., 2006, 97, 187401.

27 A. C. Ferrari and J. Robertson, Phys. Rev. B: Condens. Matter Mater. Phys., 2000, 61, 14095.

28 Z. Wen, X. Wang, S. Mao, Z. Bo, H. Kim, S. Cui, G. Lu, X. Feng and J. Chen, Adv. Mater., 2012, 24, 5610-5616.

29 M.-S. Balogun, M. Yu, C. Li, T. Zhai, Y. Liu, X. Lu and Y. Tong, J. Mater. Chem. A, 2014, 2, 10825-10829.

30 N. K. Ponon, D. J. Appleby, E. Arac, P. King, S. Ganti, K. S. Kwa and A. O'Neill, Thin Solid Films, 2015, 578, 31-37.

$31 \mathrm{~W}$. Zhang, X. Xu, C. Zhang, Z. Yu, Y. Zhou, Y. Tang, P. Wu and S. Guo, Small Methods, 2017, 1, 1700167.

32 T. Kim, G. Jung, S. Yoo, K. S. Suh and R. S. Ruoff, ACS Nano, 2013, 7, 6899-6905.

33 Y. Shao, M. F. El-Kady, L. J. Wang, Q. Zhang, Y. Li, H. Wang, M. F. Mousavi and R. B. Kaner, Chem. Soc. Rev., 2015, 44, 3639-3665.

34 B. Ahmed, D. H. Anjum, M. N. Hedhili, Y. Gogotsi and H. N. Alshareef, Nanoscale, 2016, 8, 7580-7587.

35 Z. Yang, M. Xu, Y. Liu, F. He, F. Gao, Y. Su, H. Wei and Y. Zhang, Nanoscale, 2014, 6, 1890-1895.

36 A. Achour, J. Ducros, R. Porto, M. Boujtita, E. Gautron, L. Le Brizoual, M. Djouadi and T. Brousse, Nano Energy, 2014, 7, 104-113. 